\title{
A route to explosive large-scale magnetic reconnection in a super-ion-scale current sheet
}

\author{
K. G. Tanaka ${ }^{1}$, K. Haijima ${ }^{2}$, M. Fujimoto ${ }^{1}$, and I. Shinohara ${ }^{1}$ \\ ${ }^{1}$ Japan Aerospace Exploration Agency, Kanagawa, Japan \\ ${ }^{2}$ Tokyo Institute of Technology, Tokyo, Japan
}

Received: 9 September 2008 - Revised: 24 November 2008 - Accepted: 21 December 2008 - Published: 23 January 2009

\begin{abstract}
How to trigger magnetic reconnection is one of the most interesting and important problems in space plasma physics. Recently, electron temperature anisotropy $\left(\alpha_{e o}=T_{e \perp} / T_{e \|}\right)$ at the center of a current sheet and non-local effect of the lower-hybrid drift instability (LHDI) that develops at the current sheet edges have attracted attention in this context. In addition to these effects, here we also study the effects of ion temperature anisotropy $\left(\alpha_{i o}=T_{i \perp} / T_{i \|}\right)$. Electron anisotropy effects are known to be helpless in a current sheet whose thickness is of ion-scale. In this range of current sheet thickness, the LHDI effects are shown to weaken substantially with a small increase in thickness and the obtained saturation level is too low for a large-scale reconnection to be achieved. Then we investigate whether introduction of electron and ion temperature anisotropies in the initial stage would couple with the LHDI effects to revive quick triggering of large-scale reconnection in a super-ion-scale current sheet. The results are as follows. (1) The initial electron temperature anisotropy is consumed very quickly when a number of minuscule magnetic islands (each lateral length is $1.5 \sim 3$ times the ion inertial length) form. These minuscule islands do not coalesce into a large-scale island to enable large-scale reconnection. (2) The subsequent LHDI effects disturb the current sheet filled with the small islands. This makes the triggering time scale to be accelerated substantially but does not enhance the saturation level of reconnected flux. (3) When the ion temperature anisotropy is added, it survives through the small island formation stage and makes even quicker triggering to happen when the LHDI effects set-in. Furthermore the saturation level is seen to be elevated by a factor of $\sim 2$ and large-scale reconnection is achieved only in this case. Comparison with two-dimensional simulations that exclude the LHDI effects confirms that the saturation
\end{abstract}

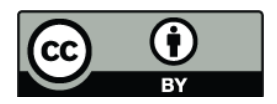

Correspondence to: K. G. Tanaka (tkentaro@stp.isas.jaxa.jp) level enhancement is due to the ion anisotropy effects, while the LHDI effects shorten the overall time scale significantly. The results imply that the ion temperature anisotropy is one of the key properties that enable large-scale magnetic reconnection to develop in a super-ion-scale current sheet.

Keywords. Space plasma physics (Magnetic reconnection; Numerical simulation studies; Waves and instabilities)

\section{Introduction}

Magnetic reconnection is one of the most import processes in space plasma physics. Magnetic reconnection converts magnetic energy to plasma kinetic and thermal energy. Its fast energy conversion rate is responsible for various explosive phenomena in the Plasma Universe (Alfvén, 1986). Magnetic reconnection is well known to be a multi-scale process. While it has macroscopic effects as a whole, its engine part is miniscule in size (electron scale) and the physics inside the engine is dominated by dynamics of electrons as collisionless charged particles under the influence of selfconsistently determined electric and magnetic fields (electron kinetic physics). Despite numerous efforts in the past, this multi-scale nature, from electron scale to MHD-scale, has been, and still is the hurdle that prevents us from fully understanding the process.

Nagai et al. (1998, 2003) showed that the quadrupolar magnetic field structure, so-called Hall-magnetic field, was detected during reconnection events in the near-Earth magnetotail by the GEOTAIL observations. The Hall structure is the evidence that the ions near the X-line are decoupled from the electron motion, that is, ion-scale physics is indeed involved in the MHD-scale process of the magnetotail that has global consequences. Recently, multi spacecraft observations by Cluster confirmed and refined the works of $\mathrm{Na}-$ gai et al. (1998). One of the clearest advantages of Cluster

Published by Copernicus Publications on behalf of the European Geosciences Union. 
observations is that the current sheet thickness during substorm events can be estimated. Nakamura et al. (2006) showed that the current sheet full thickness had a scale of the ion inertial length. Runov et al. (2008) showed that the current sheet thickness dynamically changes and estimated the current sheet thickness to be two ion inertial lengths.

The hurdle set by the multi-scale nature seems to appear highest when one considers the triggering of magnetic reconnection. The current sheet before an onset of reconnection is macroscopic, that is, its thickness is larger than the ion scale. Triggering of reconnection is to set up the minuscule engine operated by electron kinetic physics inside this macroscopic current sheet, that is, cross-scale coupling sits right at the heart of the process. The present paper will report the results from one of the dedicated studies.

In dealing with the magnetic reconnection triggering, three are three major viewpoints to evaluate the effectiveness of the process. (1) How quick is the triggering? (2) Does it lead to large enough reconnected flux such that reconnection fully across the current sheet (large-scale reconnection) is achieved? (3) Up to what thickness can it stay to be quick and large-scale? In this paper, various processes introduced below are studied and effectiveness of each is evaluated from the above points of view.

In the first place, it is worth reviewing a brief history of the tearing instability (TI) analyses and related simulation results. Coppi et al. (1966) proposed that TI has the potential of initiating magnetic reconnection. This instability produces the magnetic component across the current sheet and causes magnetic island formation. Recent 2-D studies, however, showed that effects of the instability diminish when the current sheet thickness is made larger than the electron inertial scale (Tanaka et al., 2004; Ricci et al., 2004). This means that extremely thin (electron scale) current sheet is required for TI in its original form to be an effective triggering agent. This seems to imply that TI is rather helpless in triggering magnetic reconnection in the magnetotail current sheet, where the current sheet thickness is at least of ionscale (Sergeev et al., 1990; Nakamura et al., 2006; Runov et al., 2008). In addition, presence of the magnetic normal component $B_{n}$ (Pritchett, 1994), and/or the guide field $B_{G}$ (Karimabadi et al., 2005a, b; Daughton and Karimabadi, 2005 ) is known to stabilize tearing mode in 2-D situation.

Meanwhile, a very quick formation of magnetic island in an ion-scale current sheet has been shown by recent threedimensional (3-D) full particle simulations (Horiuchi and Sato, 1999a; Lapenta and Brackbill, 2000, 2002; Scholer et al., 2003; Shinohara and Fujimoto, 2005). In contrast to the 2-D situation, in a 3-D system the lower-hybrid drift instability (LHDI) is allowed to develop at the outer edges of the current sheet (Davidson et al., 1977). The LHDI reduces the current density locally at the outer edges (Huba et al., 1980), which induces non-local redistribution of current density within the current sheet (Daughton, 2003). When the current sheet half-thickness was $D \sim 0.5 \lambda_{i}$ ( $D$ : half- thickness of the current sheet, $\lambda_{i}$ : ion inertial length), it was shown that the induced current density enhancement at the neutral sheet was strong enough to lead to vigorous growth of $\mathrm{TI}$ in the newly formed embedded current sheet. This chain of non-local coupling between the LHDI effects and the TI excitation is termed Quick Magnetic Reconnection Triggering (QMRT) (Shinohara and Fujimoto, 2005). The time scale of QMRT is comparable to the LHDI time scale. In other words, QMRT time scale is comparable to that of the fastest growing mode in the system. This type of QMRT was called Type-I in Tanaka et al. (2006).

Recent 2-D full particle simulations of the LHDI by Daughton et al. (2004) have suggested another possible type of QMRT in a thicker current sheet. It is known that the electron anisotropy $\alpha_{e}=T_{e \perp} / T_{e / /}>1$ enhances the TI growth rate significantly (e.g. Ricci et al., 2004; Karimabadi et al., 2004). Daughton et al. (2004) showed that, in a current sheet thicker than those studied by Shinohara and Fujimoto (2005), the current density redistribution induced by LHDI did not produce enhanced current layer at the neutral sheet but instead produced the electron anisotropy of $\alpha_{e}>1$. It was suggested that this anisotropy would greatly boost up TI growth rate and that QMRT of different type (Type-II) would show up. Tanaka et al. (2006) inspected if Type-II QMRT truly exists. Using the same initial settings of Shinohara and Fujimoto (2005) but with a thicker current sheet, Tanaka et al. (2006) found that the transition from Type-I to Type-II indeed occurs between $D / \lambda_{i}=0.875$ and 1 . When $D / \lambda_{i}=1$, while the current density did not enhance at the center, the magnetic walls approaching inward from the edges to the center heated the electrons anisotropically (in a manner described by Daughton, 2004) to produce the temperature anisotropy $\alpha_{e} \sim 1.2$. Insignificant as it seems, this small anisotropy actually enhanced the TI growth rate by more than an order of magnitude.

The powerfulness of the electron anisotropy effects made us revisit the study by Tanaka et al. (2004), in which a systematic survey of the TI saturation level dependence on $D$ had been made. It had been shown by 2-D simulations that there was a critical thickness $D_{c r}$, above which no significant reconnection occured. Haijima et al. (2008) investigated if the barrier set by the critical thickness could be overcome by the electron anisotropy effects. It was shown that the initial electron temperature anisotropy $\alpha_{e o}$ did not elevate the TI saturation level in a current sheet thicker than the critical thickness $D_{c r} . \alpha_{e o}$ boosted up the reconnection rate only in the very early phase resulting in the formation of minuscule magnetic islands. $\alpha_{e o}$ was consumed during this island formation phase, however, and did not assist to boost up the amount of reconnected flux later.

In addition to studying the electron anisotropy effects, Haijima et al. (2008) gave an interesting discussion on of the ion temperature anisotropy $\alpha_{i o}$. The preliminary 2-D results with $\alpha_{i o}=2$ shown in the paper suggested that, while the gain in the linear growth rate was not impressive, the suppression 
on the non-linear saturation level set by the critical thickness would be overcome by the ion anisotropy effects. Haijima et al. (2008) proposed a possible scenario of reconnection triggering in an initially thick current sheet: (1) Suppose that the magnetic field is anti-parallel across the current sheet and the current sheet is subject to thinning over a large lateral extent due to an external force. (2) At the same time as the current sheet thickness reduction, there would arise both ion and electron temperature anisotropies $\left(\alpha_{i}>1\right.$ and $\left.\alpha_{e}>1\right)$ inside the current sheet. (2) At the center of the current sheet, where the magnetic field is absent, the anisotropic electrons excite the Weibel instability (Weibel, 1959; Gary, 1993). That instability is insensitive to the initial current sheet thickness. (3) At some stage, unless the thinning is very fast, TI coupled to the Weibel instability start to grow even when the thinning current sheet thickness is still super-critical $D>D_{c r}$. (4) The ion anisotropy would help TI to have a large saturation level despite the fact that $D>D_{c r}$ and leads to macroscopic consequences.

In addition to the kinetic approaches mentioned above, previous theoretical analyses in the magneto-hydro dynamics (MHD) framework have demonstrated (Gamayunov et al., 1999) the impact of the ion pressure anisotropy on the growth rate of TI. For a typical corona situation, they concluded that admixture of hot anisotropic ions (the perpendicular temperature being larger than that of the parallel) with cold components makes TI much faster.

Here stimulated by the scenario of Haijima et al. (2008), we will make a systematic study. Various runs are performed to see how the reconnection triggering agents that have attracted recent attentions (QMRT, $\alpha_{e}$, and $\alpha_{i}$,) can collaborate with each other to gain a high saturation level quickly.

\section{Simulation setup and diagnostics}

Simulations are performed by the three-dimensional (3-D) particle-in-cell code (Shinohara and Fujimoto, 2005). The initial setting is the same as the previous studies (Tanaka et al., 2006) except that the electron temperature anisotropy is introduced. The magnetic field is given by the Harrissheet (Harris, 1962), $B_{x}(z)=B_{o x} \tanh (z / D)$, where $B_{o x}$ is the asymptotic Harris field, and $D$ is the current sheet half thickness, respectively.

The plasma number density of the current sheet is given as $n(z)=n_{C S} / \cosh ^{2}(z / D)$, where $n_{C S}$ is the plasma number density at the center of the current sheet, $z=0$. The initial ion temperature inside the current sheet is set by $T_{i, y y, C S}=T_{i, z z, C S}=T_{i, C S}$, and $T_{i, x x, C S}=T_{i, C S} / \alpha_{i o}$, where $T_{i, x x}, C S, T_{i, y y, C S}$, and $T_{i, z z, C S}$ are the diagonal components of the ion temperature tensor, respectively. $\alpha_{i o}$ denotes the initial perp-to-para temperature anisotropy of the initial current sheet ions. Likewise, for electrons, $T_{e, y y, C S}=T_{e, z z, C S}=T_{e, C S}$, and $T_{e, x x, C S}=T_{e, C S} / \alpha_{e o}$, where $\alpha_{e o}$ is the initial anisotropy. The ion-to-electron temperature ratio is set to be $T_{i, C S} / T_{e, C S}=8$. This setting is intended to model the near-Earth tail situation. In this paper, anisotropy is given by reducing the x-directional thermal velocity $v_{t h, x}$ for all of the CS particles. While we reduce $T_{j, x x}, C S$, we do not increase $T_{j, z z, C S}$ because $T_{j, x x}, C S$ reduction does nothing to the one-dimensional (z-directional) Harris equilibrium.

In addition to the current sheet component, the background plasma is added and given as $n(z)=n_{\text {lobe }}\left\{1-1 / \cosh ^{2}(z / D)\right\}$, where $n_{\text {lobe }}$ is the lobe plasma density at $|z|=\infty$. $n_{\text {lobe }}$ is set to be $n_{\text {lobe }}=0.1 \mathrm{n}_{C S}$. The background plasma temperature is isotropic, uniformly distributed, and given as $T_{i, B G}=T_{e, B G}=T_{e, C S}=0.125 T_{i, C S}$.

Hereinafter, magnetic field and density will be normalized by $B_{o x}$, and $n_{C S}$, respectively. The temporal, spatial, and velocity scales are normalized by the ion inertial length, $\lambda_{i}=c / \omega_{p i}$, the inverse of the ion gyro frequency, $\Omega_{i}^{-1}$, and the ion Alfvén velocity, $V_{A i}=B_{0 x} /\left(4 \pi M_{i} n_{C S}\right)^{0.5}$, where $c$, $\omega_{p i}, M_{i}$ are the speed of light, the ion plasma frequency, and the ion mass, respectively. The ion-to-electron mass ratio $M$ is set to be $M=M_{i} / M_{e}=400$. The electron plasma/gyro frequency ratio $\tau$ is set to be $\tau=\omega_{p e} / \Omega_{e}=1$. The electron thermal speed $v_{e}$ is set to be $v_{e}=0.33 c$. These choices reflect compromises due to limited computational resources available today.

While $B_{n}$ and/or $B_{G}$ may exist in the real situation, the present work excludes them in order to understand the reconnection triggering in the simplest situation.

The dimensions of the simulation box are $\left[0, L_{x}\right] \times[0$, $\left.L_{y}\right] \times\left[-L_{z} / 2, L_{z} / 2\right]$ with $L_{x}=12 D$ (the wavelength of the fastest growing mode (Brittnacher et al., 1995)), $L_{y}=1.0$ and $L_{z}=8 D$ in the normalized unit. The choice of $L_{y}=1.0$ excludes any longer-wave drift modes, such as the drift-kink instability (DKI), or the drift-sausage instability (DSI) (e.g. Ozaki et al., 1996; Lapenta and Brackbill, 1997; Horiuchi and Sato, 1999b), from this study. Periodic boundary conditions are imposed in both the $\mathrm{x}$ - and $\mathrm{y}$-direction, while conducting walls are set at the $\mathrm{z}$ boundary. The spatial grid of $\Delta x=\Delta y=\Delta z=0.9 \lambda_{D e}$ ( $\lambda_{D e}$ : the electron Debye length) and the time step of $\Delta t=0.15625 \Omega_{e}^{-1}$ are adopted, respectively. The number of the grids in one ion inertial length is $\lambda_{i}=64 \Delta x$. The total number of the particle is $\sim 2.0 \times 10^{9}$ for the $D=1.125$ case.

The fastest growing mode of the LHDI satisfies $\boldsymbol{k}_{\mathrm{LHD}} \cdot \boldsymbol{B} \approx 0$ at the edge of the sheet, where $\boldsymbol{k}_{\mathrm{LHD}}$ and $\boldsymbol{B}$ are the LHDI wave vector and the local magnetic field, respectively. While we do not make a non-local linear analysis to determine precisely the direction of the fastest growing LHDI wave vector, $\boldsymbol{k}_{\mathrm{LHD}} \cdot \boldsymbol{B}=0$ is a reasonable guess (Davidson et al., 1977; Hsia et al., 1979). Without a guide field, the LHDI excited at both the edges of the current sheet has the wave vector in the $y-z$ plane. On the other hand, TI grows in the $\mathrm{x}-\mathrm{z}$ plane.

This paragraph introduces some useful notations and diagnostics which will frequently appear in the following 
(a)
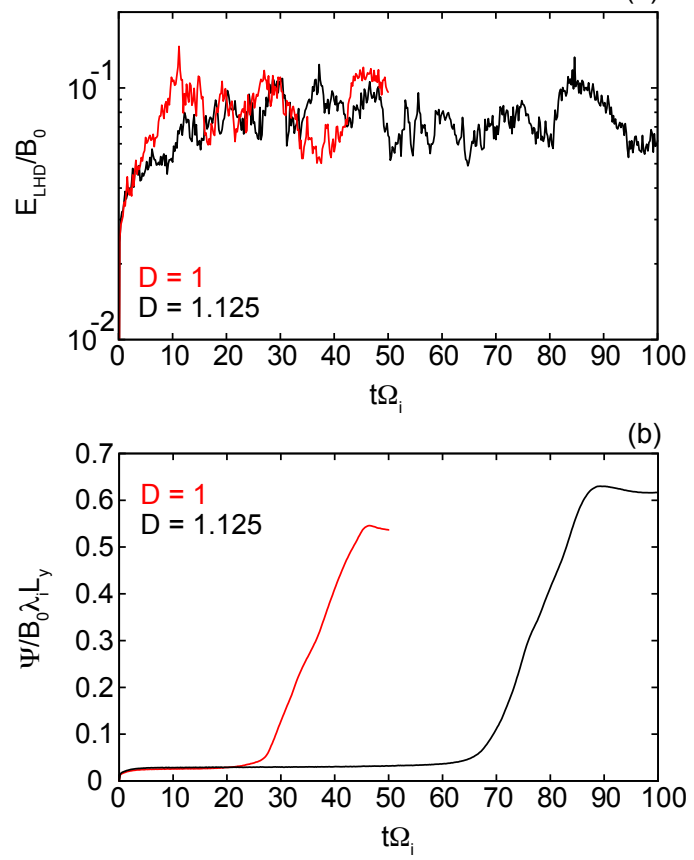

(c)

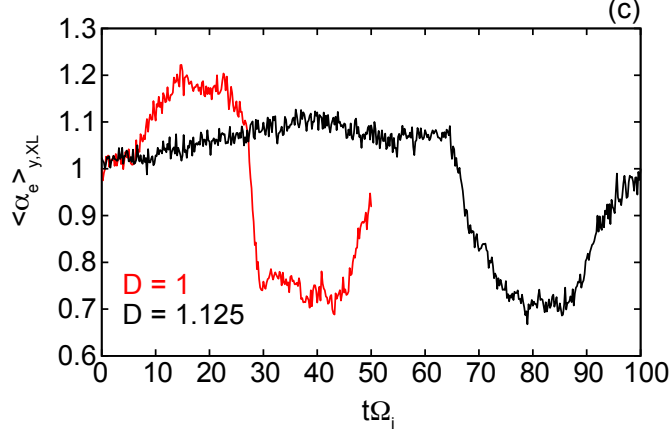

(d)

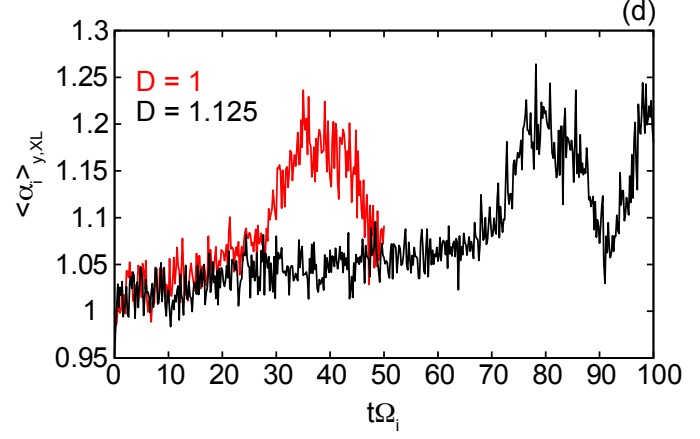

Fig. 1. Comparison between the $D=1$ and the $\mathrm{D}=1.125$ cases with $\alpha_{i o}=\alpha_{e o}=1$. Red (black) lines in panels (a), (b), (c), and (d) represent the $D=1$ (1.125) case. (a) The temporal developments of the LHDI activity $E_{\text {LHD }}$. (b) The temporal development of reconnected magnetic flux $\Psi$. (c) The temporal variation of the electron temperature anisotropy at the $\mathrm{X}$-line $<\alpha_{e}>_{y, X L}$. (d) The temporal variation of the ion temperature anisotropy at the $\mathrm{X}$-line $\left\langle\alpha_{i}\right\rangle_{y, X L}$. In both cases, explosive reconnection (starts at $t=27$ and 60 , respectively) is involved and grows in a similar manner. The problem, however, is that saturation level of $\Psi$ turns out to be rather low already at $D=1$. sections. We will observe the LHDI saturation at $z= \pm D$ (local effect). In order to see the LHDI activity, we inspect the intensity of the y-directional electric field at the edge of the current sheet $E_{\mathrm{LHD}}=\max \left(\left|E_{y}(z= \pm D)\right|\right)$. In order to measure reconnection rate and intensity, we use the reconnected magnetic flux $\Psi=0.5 \int\left|B_{z}\right| d x d y$ on the $z=0$ plane. We often average the quantities in the $\mathrm{y}$-direction at the $\mathrm{X}$-line. Here $<\ldots\rangle_{y, X L}$ denotes such spatial averaging. The temperature anisotropy $\alpha_{s}$ is introduced as $\alpha_{s}=0.5\left(T_{s, y y}+T_{s, z z}\right) / T_{s, x x}$, where $s$ stands for i (ions) or e (electrons). For instance, $\alpha_{e}=\alpha_{e 0}$ at $t=0$. In order to measure the temporal development of temperature anisotropy, we use $<\alpha_{s}>_{y, X L}$. In order to see the $\mathrm{z}$-directional spatial structure of the electron current density, we use the current density deviation from the initial value $\delta<j_{e y}>_{y, x X L}$, where $x_{X L}$ is the value of $\mathrm{x}$ at the $\mathrm{X}$-line, and $\delta$ denotes the deviation from the initial.

\section{Simulation results}

\subsection{Comparison between $D=1$ and 1.125 cases with $\alpha_{i o}=1$ and $\alpha_{e o}=1$}

Figure 1 compares the results from the $D=1$ and the $D=1.125$ cases, both with $\alpha_{i o}=1$ and $\alpha_{e o}=1$. Red (black) lines in Fig. 1a, 1, c, and d represent the $D=1$ (1.125) case. In Fig. 1a, the temporal development of $E_{\mathrm{LHD}}$ is plotted. Figure $1 \mathrm{~b}$ shows temporal development of the reconnected flux $\Psi$. Figure 1c and $\mathrm{d}$ shows the temporal variation of the electron and ion temperature anisotropy $<\alpha_{e}>_{y, X L}$, and $<\alpha_{i}>_{y, X L}$, respectively.

The $D=1$ case has been reported in detail by Tanaka et al. (2005) and only the essence is repeated here. $E_{\text {LHD }}$ achieves the saturation at $t=T_{\mathrm{LHD}}=11.2$. This LHDI activity at the edges compresses the current sheet center to produce anisotropic electrons there (Fig. 1c). The current sheet structure along the $\mathrm{z}$-axis is modified to a bifurcated profile filled with anisotropic electrons. The initial slow reconnection activity starting at $t=T_{R X}=15.8$ is due to the excitation of TI boosted by the anisotropy (Karimabadi et al., 2004). While the reconnection activity starts at $t=T_{R X}$, explosive growth begins subsequently at $t=T_{\exp }=27$ seen in Fig. 1b. At this time bifurcated current density peaks merge into a single maximum at $z=0$.

The weakened LHDI activity and thus less modification of the current sheet structure in the $D=1.125$ case results in weaker electron anisotropy $\alpha_{e}$ (peak value of 1.1 compared to 1.2 of the $D=1$ case). This weakened anisotropy makes the triggering and the development of initial reconnection phase to be much slower $\left(T_{R X}=32\right.$ and $T_{\exp }=60$ compared to 15.8 and 27 , respectively, of the $D=1$ case.). The implication is that the small increase in the current sheet thickness (merely by $\sim 10 \%$ ) can substantially (by a factor of $\sim 2$ ) delay the onset of Type-II QMRT. 

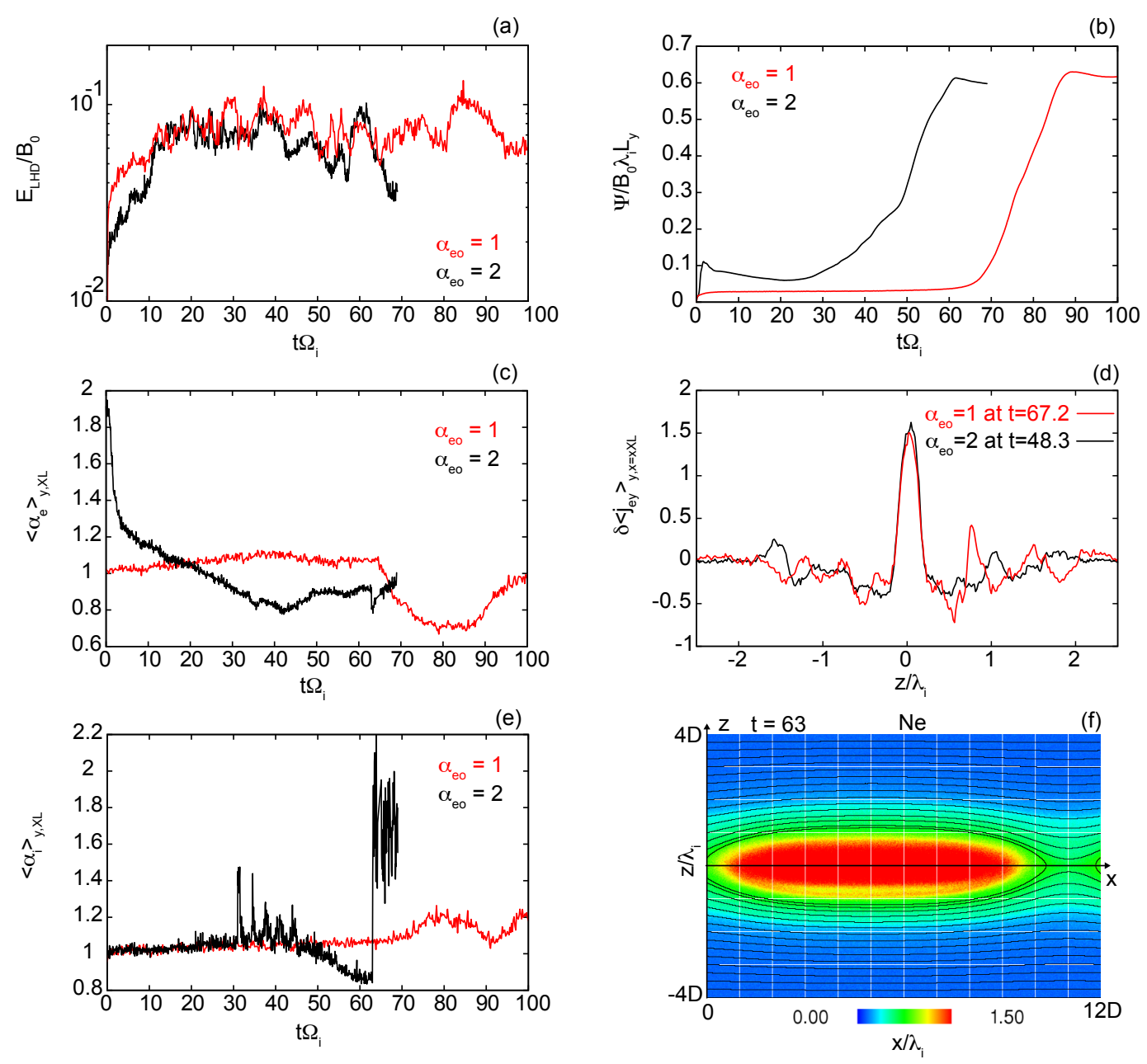

Fig. 2. Comparison between the $\alpha_{e o}=1$ and the $\alpha_{e o}=2$ cases with $D=1.125$ and $\alpha_{i o}=1$. The red (black) lines show the results of $\alpha_{e o}=1$ (2) case, respectively. The temporal developments of (a) $E_{\mathrm{LHD}}$, (b) $\Psi$, (c) $<a_{e}>_{y, X L}$, and (e) $<\alpha_{i}>_{y, X L}$ are shown. (d) $\delta<j_{e y}>y, x X L$ along $z$ at the onsets of explosive reconnection. (f) 2-D XZ contour of plasma number density (color) and the field lines (black curve) at the saturation time of $t=63$ from the $\alpha_{e o}=2$ case. It is found that field line reconnection fully across the current sheet is failed to occur even with the aid of the $\alpha_{e o}$ effects. The electron anisotropy effects do not save the QMRT from the crisis of being not able to trigger a large-scale reconnection.

Comparison between Fig. 1a and c shows that when the explosive reconnection starts, the electron anisotropy suddenly falls down to below unity. On the other hand, Fig. 1d shows that the ion anisotropy lifts off and saturates at $t=40$ and 80 for $D=1$ and 1.125 cases, respectively, at the same saturation level of $\alpha_{i} \sim 1.2$.

The difference between $\alpha_{i}$ and $\alpha_{e}$ in those temporal developments can be understood by their inflow and outflow patterns. Until the explosive reconnection phase, the electron temperature anisotropy increases in reaction with the nonlinear effect of the LHDI. During this phase, the ions are isotropic because their inflows to the current sheet are insignificant. Once the explosive reconnection takes place, the ions flow into the X-line from above and below to the $z=0$ plane in association with the current sheet crash. At the same time, the electron streaming towards the X-line but along the field lines occurs.

In Fig. 1b, while the time that takes for the explosive reconnection to happen is different, the growth curves of $\Psi$ are the same after the onset of explosive phase. The saturation level is also the same and turns out to be rather low. Indeed at saturation the plasma density is modest at the X-line (not shown) indicating that the lobe magnetic field lines do not undergo reconnection in these settings. The interval of the explosive phase is so short that it terminates before field line reconnection fully across the current sheet occurs. In other words, one may say that large-scale reconnection is achieved in neither of the isotropic cases with $D \geq 1$. In this context, QMRT with isotropic plasma has the trouble already at $D=1$. 

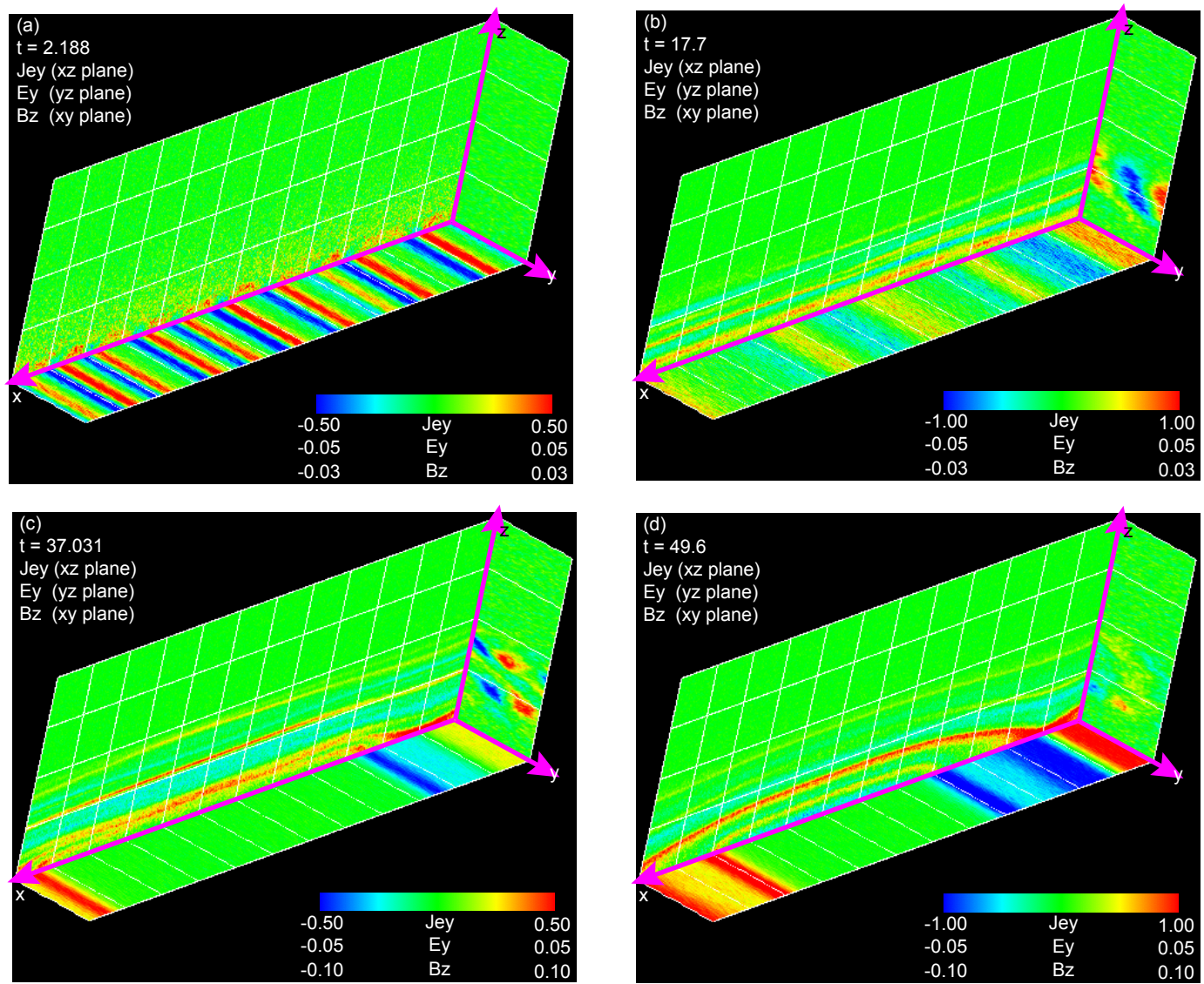

Fig. 3. 3-D snapshots from the $\alpha_{i o}=1, \alpha_{e o}=2$, and $D=1.125$ case. (a) $t=2.188$, (b) 17.7 , (c) 37.1 , and (d) 49.6. Color contours on the XY, $\mathrm{YZ}$, and XZ planes are for $B_{z}, E_{y}$, and $j_{e y}$, respectively. The displayed domain is $0<x<12 D, 0<y<1$, and $0<z<4 D$. In order to see the $\mathrm{y}$-directional structure easily, elongation by a factor of 4 in the $y$-direction is applied. See text for details.

\subsection{Comparison between $\alpha_{e o}=1$ and 2 cases at $D=1.125$ $\left(\alpha_{i o}=\mathbf{1}\right)$}

Since the weaker activity in the $D=1.125$ case shown above is due to smaller electron anisotropy induced by the weaker LHDI activity, investigation of the effects of the anisotropy given in the initial condition should be interesting. Figure 2 compares the results between $\alpha_{e o}=1$ and 2 cases with $D=1.125$ and $\alpha_{i o}=1$. The red (black) lines show the results of $\alpha_{e o}=1$ (2) case, respectively. As for $E_{\mathrm{LHD}}$ is concerned there is little difference between the two cases. In contrast, Comparison of $\Psi$ (Fig. 2b) shows quite different histories. During $t=0 \sim 2$, an abrupt jump is observed in the anisotropic case. This reflects quick formation of magnetic islands of small wavelength, which is in accordance with the linear theory (Karimabadi et al., 2004). Note, however, that significant reduction of $\alpha_{e}$ results from the x-directional electron heating in the island formation process in this very early phase (Fig. 2c). That short time-scale drop of $\alpha_{e}$ agrees well with the previous report by Ricci et al. (2004). That sharp drop of $\alpha_{e}$ in the early stage of the $\alpha_{e o}=2$ case is likely to be coupled to the electron Weibel instability (Weibel, 1959; Gary, 1993;
Gary and Karimabadi, 2006), which was pointed out by Haijima et al. (2008). Until $t=2$, the observed fastest growing mode on the $z=0$ plane has $\lambda=1.5 \sim 3$ (we term these to be minuscule islands).

Then the reconnected flux decreases until $t \sim 25$. During this interval magnetic loops that form the minuscule islands are re-opening without, unlike in a case where dynamic coalescence of magnetic islands leads to formation of a single dominant $\mathrm{X}$-line, activating some selected $\mathrm{X}$-lines to produce more reconnected flux than re-opened (see also Fig. 3b). Around $t \sim 25 \Psi$ turns to increase with an enhancement of $\alpha_{i}$ (Fig. 2e). Anatomy of the reconnected flux reveals three stages in the anisotropic case: (1) minuscule magnetic island phase due to electron anisotropy $(t<25),(2)$ slow reconnection phase $(t=25 \sim 50)$, and then, (3) explosive reconnection phase $(t=50 \sim 60)$.

Figure $2 \mathrm{~d}$ compares $\delta<j_{e y}>_{y, x X L}$ upon the start of the explosive phase. In this figure, the red (black) line is the $\alpha_{e o}=1$ (2) case at $t=67.2$ (48.3). This figure shows that the $\alpha_{e o}=2$ case makes current sheet thickness down to an electron-scale quicker than the $\alpha_{e o}=1$ case does. 
Figure $2 \mathrm{f}$ shows 2-D XZ contour of plasma number density at the saturation time of $t=63$ from the $\alpha_{e o}=2$ case. In this figure, the black curves are the field lines. The modest density at the $\mathrm{X}$-line, not as low as in the lobe region, shows that no lobe-reconnection is achieved in this case. That is, field line reconnection fully across the current sheet is failed to occur even with the aid of the $\alpha_{e o}$ effects. The electron anisotropy effects do not save the QMRT from the crisis of being not able to trigger a large-scale reconnection.

Figure 3 shows three-dimensional snapshots of the results from the $\alpha_{e o}=2$ case. Four Panels are at $t=2.188,17.7,37.1$, and 49.6, respectively. In these figures, color contours on the $\mathrm{XY}, \mathrm{YZ}$, and XZ planes are for $B_{z}, E_{y}$, and $j_{e y}$, respectively. The displayed domain is $0<x<12 D, 0<y<1$, and $0<z<4 D$. In order to see the $y$-directional structure easily, elongation by a factor of 4 in the $y$-direction is applied. At $t=2.188$, multiple pairs of positive/negative $B_{z}$ are found on the XY plane (Fig. 3a). That is, the initial electron anisotropy produces multiple minuscule magnetic islands. At $t=17.7, E_{y}$ disturbances on the YZ plane due to LHDI are clearly seen. $B_{z}$ on the $X Y$ plane shows smaller amplitude and larger wavelength feature than at $t=2.188$, indicating that re-opening of small closed magnetic loops have taken place. Figure $3 \mathrm{c}$ shows the structure at $t=37.1$, which is during the time of slow reconnection growth (Fig. 2b). While an enhanced $j_{e y}$ is created at the $\mathrm{X}$-line $(x=1.125)$, other two enhanced $j_{e y}$ are also seen at $x=2.812$, and $x=12.94$ on the XZ plane. At these two locations, local peaks of $B_{z}$ are seen on the XY plane. This $j_{e y} / B_{z}$ structure is maintained between $t=25 \sim 50$ when the ion anisotropy shows spiky enhancements (Fig. 2e) and when the reconnected flux shows slower increase (Fig. 2b). Finally at $t=49.6$ a strong $j_{e y}$ concentration is seen to dominate at the $\mathrm{X}$-line (the $\mathrm{XZ}$ plane). This opens the time interval when reconnection proceeds explosively (Fig. $2 b$ ).

\subsection{Comparison between $\alpha_{i o}=1$ and 2 at $D=1.125$ with $\alpha_{e o}=2$}

Now we add the ion anisotropy and study its effect. Figure 4 compares the $\alpha_{i o}=1$ and 2 cases with $D=1.125$ and $\alpha_{e o}=2$. In Fig. $4 a, E_{\text {LHD }}$ of the two cases show almost the same temporal development until the end of the simulation. Figure $4 \mathrm{~b}$ compares $\Psi$. Both cases with the electron temperature anisotropy show a three-stage feature, which for the $\alpha_{i o}=2$ case is described as follows: (1) minuscule magnetic island phase due to electron anisotropy $(t<23)$, (2) slow reconnection phase ( $t=23 \sim 32)$, and (3) explosive reconnection phase $(t=32 \sim 45)$. The slow reconnection phase is characterized by the fall-down of $\alpha_{e}$ to unity (Fig. 4c). For both cases in Fig. 4c, the sharp drop of $\alpha_{e}$ is also seen in the early stage, which was already seen in Fig. 2c. On the other hand, the onset of the explosive phase is characterized by the elevation of $\alpha_{i}$ (Fig. 4d) for the $\alpha_{i o}=2$ case $(T=32)$ while it starts to be reduced for the other case $(T=50)$. In Fig. $4 \mathrm{~b}$, the explosive reconnection saturates at $t=45$ with $\Psi=1.4$, which is doubled
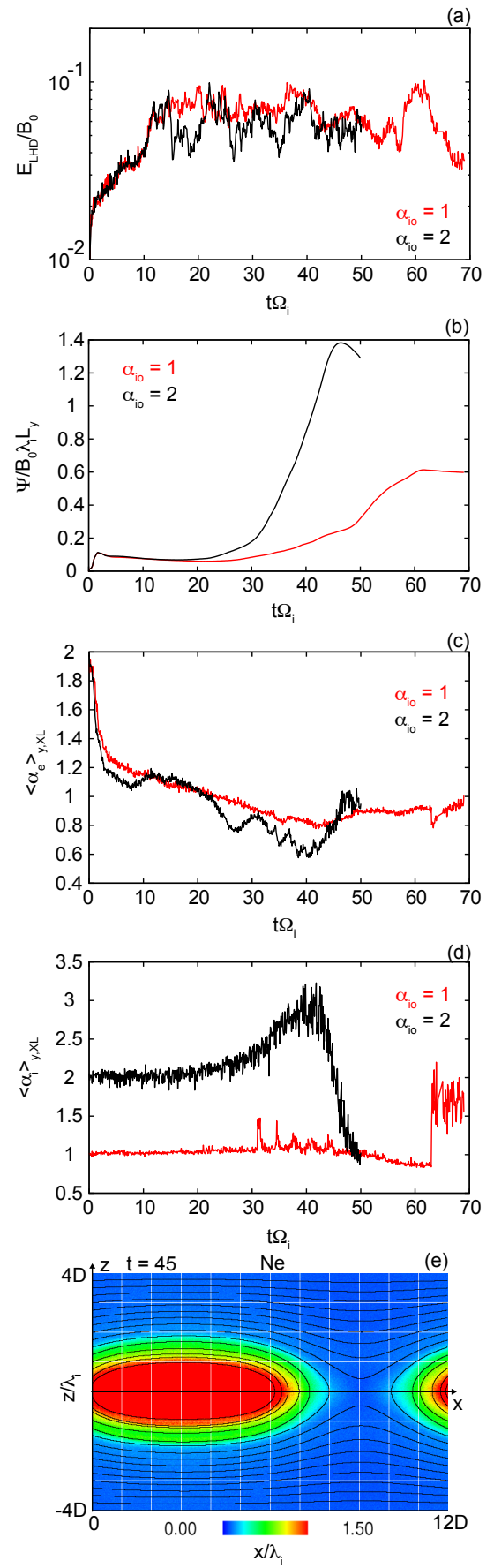

Fig. 4. Comparison between the $\alpha_{i o}=1$ and the $\alpha_{i o}=2$ cases with $D=1.125$ and $\alpha_{e o}=2$. The red (black) lines show the results of $\alpha_{i o}=1$ (2) case, respectively. Temporal developments of (a) $E_{\mathrm{LHD}}$, (b) $\Psi$, (c) $<a_{e}>_{y, X L}$, and (d) $<\alpha_{i}>_{y, X L}$ are shown. (e) 2-D XZ contour of plasma number density (color) and the field lines (black curve) at the saturation time of $t=45$ from the $\alpha_{i o}=2$ case. With $\alpha_{i o}=2$, the explosive reconnection saturates at $t=45$ with $\Psi=1.4$, which is doubled compared to the $\alpha_{i}=1$ case. At the same time, 2-D XZ contour shows that field line reconnection fully across the current sheet is attained with the help from the ion anisotropy effects. 

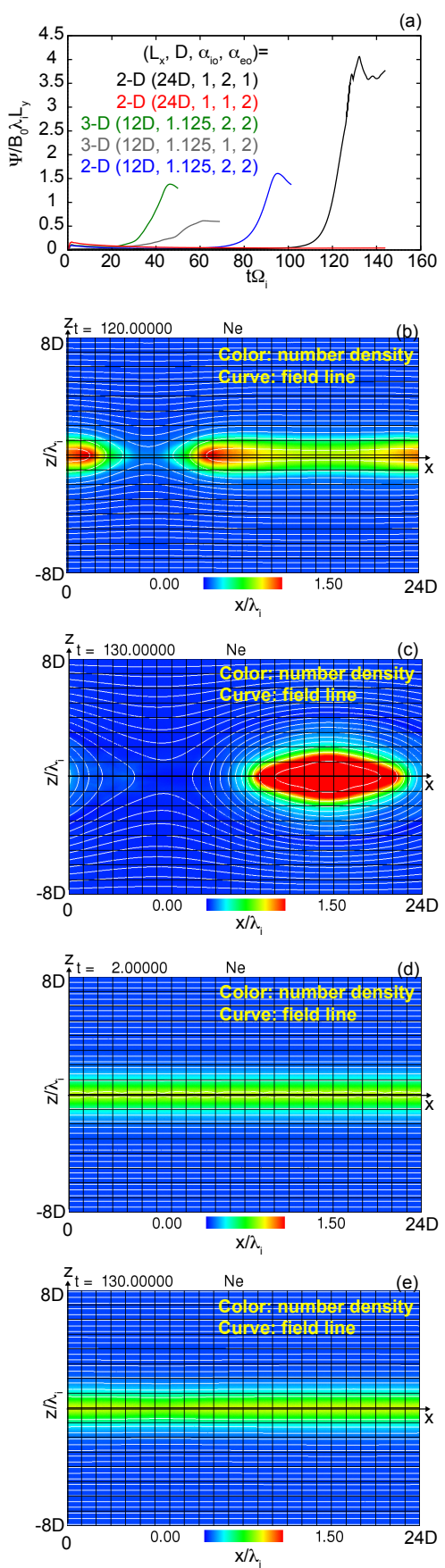

Fig. 5. Effects of the ion temperature anisotropy on the saturation level. (a) The temporal developments of reconnected flux $\Psi$ from various runs. (b) and (c) 2-D XZ contours of plasma number density (color) and the field lines (black curve) at $t=120$ and 130, respectively, from the 2-D $\left(L_{x}, D, \alpha_{i o}, \alpha_{e o}\right)=(24 D, 1,2,1)$ case. $(\mathbf{d})$ and (e) 2-D XZ contours of plasma number density (color) and the field lines (black curve) at $t=2$ and 130, respectively, from the 2-D ( $L_{x}$, $\left.D, \alpha_{i o}, \alpha_{e o}\right)=(24 D, 1,2,1)$ case. The runs showing high saturation level of $\Psi$, as high as to attain reconnection fully across the current sheet, are all with $\alpha_{i o}=2$. compared to the $\alpha_{i o}=1$ case. This difference in the reconnected flux produces qualitative difference in the magnetic island configuration. Figure $4 \mathrm{e}$ shows $2-\mathrm{D} \mathrm{XZ}$ contour of plasma number density at the saturation time of $t=45$. In this figure, the black curves are the field lines. The density at the $\mathrm{X}$-line being as low as in the lobe region is the evidence that lobe-reconnection is achieved in this case. That is, field line reconnection fully across the current sheet is attained with the help from the ion anisotropy effects.

\section{Discussion}

\subsection{Summary}

In the present study, we have carried out a series of threedimensional (3-D) particle-in-cell simulations to investigate how the three agents that attracted recent attention collaborate in triggering reconnection in a super-ion scale current sheet.

At $D=1$ with $\alpha_{e o}=1$, quick restructuring of the current layer structure takes place to form a bifurcated current layer in reaction to the lower-hybrid drift instability (LHDI). Associated with the current sheet modification, the electron temperature anisotropy at the center of current sheet develops up to $\alpha_{e}=1.2$, which enables the magnetic reconnection to be easily triggered. In contrast, at $D=1.125$ with $\alpha_{e o}=1$, less enhancement of the electron anisotropy at $z=0$ results in much slower QMRT. In either case the saturation level is so low that magnetic reconnection fully across the current sheet is not achieved. These results show the limitation of the TypeII QMRT process in the super ion-scale thickness regime.

In order to examine if the initial electron temperature anisotropy revives the QMRT at $D=1.125$, we artificially set $\alpha_{e o}$ to be 2 . The results have shown that the initial high $\alpha_{e o}$ is relaxed quickly by the electron Weibel instability in the early time during which the formation of multiple islands with $\lambda \sim 1.5$ to 3 heats the electrons in the parallel direction. The results are fairly in good agreement with Haijima et al. (2008). Since the electrons quickly become isotropic before QMRT sets-in, the initial electron anisotropy is not very effective in assisting QMRT. While the time scale is shortened, the problem of low saturation level remains.

We then proceed to add the effect of ion temperature anisotropy. $\alpha_{i o}$ is maintained during the small island formation phase until the onset of the explosive reconnection. In association with the explosive flux growth, $\alpha_{i}$ increases. The ion anisotropy effects double the saturation level of the reconnected flux and a large-scale reconnection is achieved only in this case.

\subsection{2-D studies in the ion anisotropy effects}

The results of the case when the ion temperature anisotropy is added show that the saturation level can be elevated significantly by this effect. Since it should be interesting to 
investigate more on the ion anisotropy effects, we have carried out additional 2-D runs. Figure 5a shows the temporal developments of reconnected flux from various runs.

The profile for the 2-D counterpart to the 3-D case with the ion anisotropy, the 2-D $\left(L_{x}, D, \alpha_{i o}, \alpha_{e o}\right)=(12 D, 1.125,2,2)$ case, is shown by the blue line. One can see that the saturation level is as high as the 3-D case (shown by the green line) indicating that the LHDI effects are not necessary to raise the saturation level. On the other hand, the time scale for the 2-D case is longer than its 3-D counterpart showing that the LHDI effects are effective in shortening the time scale.

The frequency ratio $\tau$ has been set to be $\tau=1$ in this paper, however, $\tau$ is typically 4 in the magnetotail, and is much larger in the magnetopause. According to recent twodimensional LHDI study (Tanaka et al., 2006), it is predicted that larger $\tau$ make the LHDI effects to be less significant. This issue makes the position of the ion anisotropy effect to be more important in considering magnetic reconnection triggering.

The effects of the ion anisotropy are shown more clearly by the profile from the 2-D $\left(L_{x}, D, \alpha_{i o}, \alpha_{e o}\right)=(24 D, 1,2,1)$ case depicted by the solid black line. The reconnected flux is doubled ( $\sim 4$ versus $\sim 1.5)$ when the system size is doubled. What we see in the $L_{x}=24 D$ case is the saturation of two magnetic island at $t=120$ (Fig. 5b) and subsequent coalescence of these two into one large island that saturates at $t=130$ (Fig. 5c). The density at the $\mathrm{X}$-lines at $t=120$ shows that lobe reconnection is already involved upon the saturation of smaller two islands. The subsequent coalescence is a dynamic one that reconnects more flux upon the merger of the two.

This should be contrasted with the results from the 2-D $\left(L_{x}, D, \alpha_{i o}, \alpha_{e o}\right)=(24 D, 1,1,2)$ case (red). Without the ion anisotropy and the LHDI effects, the electron anisotropy effect alone in the ion-scale current sheet is so helpless as to produce negligible reconnected flux. While many minuscule islands are formed at $t=2$ (Fig. 5d), subsequent interaction among the islands are more or less only to diffuse the current sheet structure (Fig. 5e: $t=130$ ) and does not lead to a dynamic formation of one large island containing reconnected ex-lobe field lines.

\subsection{What generates $\alpha_{i o}$ ?}

One plausible mechanism to generate ion anisotropy is seen in recent particle simulations of Lapenta and King (2007). In their simulations, an initially uniform plasma sheet is pushed at its boundary. The compression generates a shock front which propagates into the current sheet center. The shock eventually leads to formation of a bifurcated current layer. During that period, the shock heats both the ion and electron perpendicular to the magnetic field at the current sheet center.

Only when a substantial ion anisotropy $\left(\alpha_{i o}=2\right.$ in the present case) is present in the current sheet, saturation level of $\Psi$ is expected to be large enough to trigger lobe reconnec- tion in an ion-scale current sheet. The anisotrpic ion heating during current sheet thinning seems to be a key issue in considering the triggering problem and deserves further studies in addition to Lapenta and King (2007).

\subsection{Notes on future works}

A nicely developed magnetic island has the horizontal/vertical aspect ratio of $\sim 2$. Then when an island having the wavelength of $\sim 12 D$ is nicely developed, since the vertical half-thickness is $\sim 3 D$, one would expect that the lobe reconnection has taken place. When the system size is made larger, this matured island will be subject to a dynamic coalescence process that increases the amount of reconnected flux. This is what we see in the 2-D $\left(L_{x}, D, \alpha_{i o}, \alpha_{e o}\right)=(24 D$, $1,2,1)$ case shown in Fig. 5. In contrast, what we see in the 2-D $\left(L_{x}, D, \alpha_{i o}, \alpha_{e o}\right)=(24 D, 1,1,2)$ case is that, if the islands subject to coalescence is very weak, the coalescence does not lead to increase the reconnected flux (This curious behavior is confirmed to be true even when the number of super-particles per cell is increased by an order of magnitude.). While the difference in these two final states seems trivial because the contrast in the status of the islands prior to coalescence is so drastic, it does introduce one to the question: Where is the line demarcating the two final states?

Our conjecture is that, if lobe reconnection is not involved upon the saturation of the island with the wavelength of $\sim 12 D$, subsequent coalescence is not dynamic and reconnected flux stops increasing. That is, it does not lead to triggering of large-scale reconnection (reconnection fully across the current sheet) however large the system size is made. In other words, one can tell whether the successful goal can be reached or not by inspecting the saturation level of the island having the wavelength of $\sim 12 D$. For instance, our prediction for the 3-D $\left(L_{x}, D, \alpha_{i o}, \alpha_{e o}\right)=(24 D, 1.125,1,2)$ case $($ when the system size $L_{x}$ is doubled to include two of what is shown in Fig. 2f) is that dynamic coalescence will not achieved and large-scale reconnection will not be triggered (Note that lobe reconnection is not seen in Fig. $2 \mathrm{f}$ where $L_{x}$ is set to $12 D$.). Indeed this is something that can be studied as soon as the next new machine arrives at JSS/JAXA and is on the top of our to-do list.

Previous studies (e.g. Pellat et al., 1991; Pritchett, 1994) showed that a small $B_{n}$ has substantial effects on the tearing mode. Whether the ion-anisotropy issue emerging here applies in the presence of non-zero $B_{n}$ is to be investigated in the future.

Acknowledgements. Numerical simulations are performed on NEC SX6 at JSS/JAXA. K. T. was supported by the Grant-in-Aid for Creative Scientific Research "The Basic Study of Space Weather Prediction" (17GS0208, Head Investigator: K. Shibata) from the Ministry of Education, Science, Sports, Technology, and Culture of Japan. K. T. was also supported by Grant-in-Aid for Japan Society for the Promotion of Science (JSPS) Fellows (20.9859). 
Topical Editor R. Nakamura thanks two anonymous referees for their help in evaluating this paper.

\section{References}

Alfvén, H.: The Plasma Universe, Phys. Today, 39, 22-27, doi:10.1063/1.88103, 1986.

Brittnacher, M., Quest, K. B., and Karimabadi, H.: A new approach to the linear theory of single-species tearing in two-dimensional quasi-neutral sheets, J. Geophys. Res., 100, 3551-3562, 1995.

Coppi, B., Laval, G., and Pelat, R.: Dynamics of the geomagnetic tail, Phys. Rev. Lett., 16, 1207-1210, 1966.

Daughton, W.: Electromagnetic Properties of the Lower-Hybrid Drift Instability in a Thin Current Sheet, Phys. Plasmas, 10, 3103-3119, 2003.

Daughton, W., Lapenta, G., and Ricci, P.: Nonlinear Evolution of the Lower-Hybrid Drift Instability in a Current Sheet, Phys. Rev. Lett., 93, 105004, doi:10.1103/PhysRevLett.93.105004, 2004.

Daughton, W. and Karimabadi, H.: Kinetic theory of collisionless tearing at the magnetopause, J. Geophys. Res., 110, A03217, doi:10.1029/2004JA010751, 2005.

Davidson, R. C., Gladd, N. T., Wu, C. S., and Huba, J. D.: Effects of finite plasma beta on the lower-hybrid-drift instability, Phys. Fluids, 20, 301-310, 1977.

Gamayunov, K. V., Oraevsky, V. N., and Kuznetsov, V. D.: Magnetic reconnection in the neutral current sheet in the presence of a small fraction of hot anisotropic ions, Plasma Phys. Control. Fusion, 40, 1285-1312, 1998.

Gary, S. P.: Theory of Space Plasma Microinstabilities, Cambridge University Press, Cambridge, 1993.

Gary, S. P. and Karimabadi, H.: Linear theory of electron temperature anisotropy instabilities: Whistler, mirror, and Weibel, J. Geophys. Res., 111, A11224, doi:10.1029/2006JA011764, 2006.

Haijima, K., Tanaka, K. G., Fujimoto, M., and Shinohara, I.: Electron temperature anisotropy effects on tearing mode in ion-scale current sheets, Adv. Space Res., 41, 1643-1648, doi:10.1016/j.asr.2007.05.049, 2008.

Harris, E. G.: On a Plasma Sheath Separation Regions of Oppositely Directed Magnetic Field, Nuovo Cimento, 23, 115-121, 1962.

Horiuchi, R. and Sato, T.: Three-dimensional particle simulation of plasma instabilities and collisionless reconnection in a current sheet, Phys. Plasmas, 6, 4565-4574, 1999a.

Horiuchi, R. and Sato, T.: Particle simulation of collisionless reconnection in three dimensions, Plasma Phys. Controlled Fusion, 41, A477-A485, doi:10.1088/0741-3335/41/3A/041, 1999b.

Hsia, J. B., Chiu, S. M., Hsia, M. F., Chou, R. L., and Wu, C. S.: Generalized lower-hybrid-drift instability, Phys. Fluid, 22, 17371746, 1979.

Huba, J. D., Drake, J. F., and Gladd, N. T.: Lower-hybrid-drift instability in field reversal plasmas, Phys. Fluids, 23, 552-561, 1980.

Karimabadi, H., Daughton, W., and Quest, K. B.: Role of temperature anisotropy in the onset of magnetic reconnection, Geophys. Res. Lett., 31, L18801, doi:10.1029/2004GL020791, 2004.

Karimabadi, H., Daughton, W., and Quest, K. B.: Antiparallel versus component merging at the magnetopause: Current bifurcation and intermittent reconnection, J. Geophys. Res., 110, A03213, doi:10.1029/2004JA010750, 2005a.
Karimabadi, H., Daughton, W., and Quest, K. B.: Physics of saturation of collisionless tearing mode as a function of guide field, J. Geophys. Res., 110, A03214, doi:10.1029/2004JA010749, 2005b.

Lapenta, G. and Brackbill, J. U.: A kinetic theory for the drift-kink instability, J. Geophys. Res., 102(A12), 27099-27108, 1997.

Lapenta, G. and Brackbill, J. U.: 3D reconnection due to oblique modes: a simulation of Harris current sheets, Nonlin. Processes Geophys., 7, 151-158, 2000,

http://www.nonlin-processes-geophys.net/7/151/2000/.

Lapenta, G. and Brackbill, J. U.: Nonlinear evolution of the lower hybrid drift instability: Current sheet thinning and kinking, Phys. Plasmas, 9, 1544-1554, doi:10.1063/1.1464149, 2002.

Lapenta, G. and King, J.: Study of current intensification by compression in the Earth magnetotail, J. Geophys. Res., 112, A12204, doi:10.1029/2007JA012527, 2007.

Nagai, T., Fujimoto, M., Saito, Y., Machida, S., Terasawa, T., Nakamura, R., Yamamoto, T., Mukai, T., Nishida, A., and Kokubun, S.: Structure and dynamics of magnetic reconnection for substorm onsets with Geotail observations, J. Geophys. Res., 103, 4419-4440, 1998.

Nagai, T., Shinohara, I., Fujimoto, M., Machida, S., Nakamura, R., Saito, Y., and Mukai, T.: Structure of the Hall current system in the vicinity of the magnetic reconnection site, J. Geophys. Res., 108(A10), 1357, doi:10.1029/2003JA009900, 2003.

Nakamura, R., Baumjohann, W., Asano, Y., Runov, A., Balogh, A., Owen, C. J., Fazakerley, A. N., Fujimoto, M., Klecker, B., and Rème, H.: Dynamics of thin current sheets associated with magnetotail reconnection, J. Geophys. Res., 111, A11206, doi:10.1029/2006JA011706, 2006.

Ozaki, M., Sato, T., Horiuchi, R., and Complexity Simulation Group: Electromagnetic instability and anomalous resistivity in a magnetic neutral sheet, Phys. Plasmas, 3, 2265-2274, doi:10.1063/1.871908, 1996.

Pellat, R., Coroniti, F. V., and Pritchett, P. L.: Does ion tearing exist?, Geophys. Res. Lett., 18, 143-146, 1991.

Pritchett, P. L.: Effect of electron dynamics on collisionless reconnection in two-dimensional magnetotail equilibria, J. Geophys. Res., 99, 5935-5941, 1994.

Pritchett, P. L.: Externally driven magnetic reconnection in the presence of a normal magnetic field, J. Geophys. Res., 110, A05209, doi:10.1029/2004JA010948, 2005.

Ricci, P., Brackbill, J. U., Daughton, W., and Lapenta, G.: Influence of the lower hybrid drift instability on the onset of magnetic reconnection, Phys. Plasma, 11, 4489-4500, doi:10.1063/1.871908, 2004.

Runov, A., Baumjohann, W., Nakamura, R., Sergeev, V. A., Amm, O., Frey, H., Alexeev, I., Fazakerley, A. N., Owen, C. J., Lucek, E., André, M., Vaivads, A., Dandouras, I., and Klecker, B.: Observations of an active thin current sheet, J. Geophys. Res., 113, A07S27, doi:10.1029/2007JA012685, 2008.

Scholer, M., Sidorenko, I., Jaroschek, C. H., Treumann, R. A., and Zeiler, A.: Onset of collisionless magnetic reconnection in thin current sheet: Three-dimensional particle simulations, Phys. Plasmas, 10, 3521-3527, 2003.

Sergeev, V. A., Tanskanen, P., Mursula, K. Korth, A., and Elphic, R.: Current sheet thickness in the near-Earth plasma sheet during substorm growth phase, J. Geophys. Res., 95, 3819-3828, 1990.

Shinohara, I. and Fujimoto, M.: Formation of thin electron current 
layer associated with lower hybrid drift instability and its relation to quick reconnection triggering, in Frontiers of magnetospheric physics, COSPAR colloquium Ser., 16, 2005.

Tanaka, K. G., Shinohara, I., and Fujimoto, M.: Critical thickness for two-dimensional tearing instability, Geophys. Res. Lett., 31, L03808, doi:10.1029/2003GL018955, 2004.

Tanaka, K. G., Shinohara, I., and Fujimoto, M.: Quick magnetic reconnection triggering in an ion-scale current sheet: Transition from Type-I to Type-II, Geophys. Res. Lett., 32, L17106, doi:10.1029/2005GL023718, 2005.
Tanaka, K. G., Shinohara, I., and Fujimoto, M.: Parameter dependence of quick magnetic reconnection triggering: A survey study using two-dimensional simulations, J. Geophys. Res., 111, A11S18, doi:10.1029/2006JA011968, 2006.

Weibel, E. S.: Spontaneously growing transverse waves in a plasma due to an anisotropic velocity distribution, Phys. Rev. Lett., 2, 83-84, 1959. 